Mathematical Modelling and Analysis

Volume 16 Number 2, June 2011, 220-232

Doi:10.3846/13926292.2011.578677

(C) Vilnius Gediminas Technical University, 2011
www.tandf.co.uk/journals/TMMA

Publisher: Taylor\&Francis and VGTU

Online ISSN: 1648-3510

Print ISSN: 1392-6292

\title{
Method of Lines and Finite Difference Schemes with the Exact Spectrum for Solution the Hyperbolic Heat Conduction Equation*
}

\author{
Harijs Kalis $^{a, b}$ and Andris Buikis ${ }^{a, b}$ \\ ${ }^{a}$ Institute of Mathematics and Computer Science, University of Latvia \\ Raina bulvāris 29, LV-1459 R̄̄ga, Latvija \\ ${ }^{b}$ Faculty of Physics and Mathematics, University of Latvia \\ Zellu ielā 8, LV-1002 Rīga, Latvija \\ E-mail(corresp.): kalis@lanet.lv \\ E-mail: buikis@latnet.lv
}

Received September 5, 2010; revised March 21, 2011; published online May 1, 2011

\begin{abstract}
This paper is concerning with the 1-D initial-boundary value problem for the hyperbolic heat conduction equation. Numerical solutions are obtained using two discretizations methods - the finite difference scheme (FDS) and the difference scheme with the exact spectrum (FDSES). Hyperbolic heat conduction problem with boundary conditions of the third kind is solved by the spectral method. Method of lines and the Fourier method are considered for the time discretization.

Finite difference schemes with central difference and exact spectrum are analyzed. A novel method for solving the discrete spectral problem is used. Special matrix with orthonormal eigenvectors is formed. Numerical results are obtained for steel quenching problem in the plate and in the sphere with holes. The hyperbolic heat conduction problem in the sphere with holes is reduced to the problem in the plate. Some examples and numerical results for two typical problems related to hyperbolic heat conduction equation are presented.
\end{abstract}

Keywords: Spectral problems, hyperbolic heat conducting equation, finite differences.

AMS Subject Classification: 65M06; 65M20; 65M70; 65N06; 65N25.

\section{Introduction}

Problems involving the hyperbolic heat conduction equation arise in modelling intensive steel quenching, laser pulse duration and other processes [2, 4]. This paper is concerning with the corresponding 1-D initial-boundary value problem. Analytical and numerical solutions are obtained using the finite difference scheme (FDS) and a difference scheme with the exact spectrum (FDSES) [5].

\footnotetext{
* Authors thank for a partial support Latvian Science Foundation grant Nr. 09.1572 and the ESF project Nr. 2009/0223/1DP/1.1.1.2.0/09/APIA/VIAA/008
} 
The second order differential operator in space is approximated by a secondorder FDS on the uniform grid. We employ the method of lines for the discretization in time and solve the corresponding equations by continuous and discrete Fourier methods. The discrete spectral method leads to a transcendent equation for the eigenvalues of the FDS. We derive a new transcendent equation to compute the two largest eigenvalues and the corresponding eigenvectors.

We find that the number of distinct eigenvalues depends on a special parameter $Q=L \sigma_{1} \sigma_{2} /\left(\sigma_{1}+\sigma_{2}\right)$, where $\sigma_{1}, \sigma_{2}$ are the heat transfer coefficients in the boundary conditions and $L$ is the length of the interval.

We define the difference scheme with the exact spectrum (FDSES) as follows. Using the eigenvalues $D$ and eigenvectors $P$ of the finite difference matrix $A$, we write the Jordan form of the matrix $A=P D P^{T}$ and replace the discrete eigenvalues on the diagonal of $D$ with the first eigenvalues of the differential operator.

Some applications are presented in Section 6. Two problems for the hyperbolic heat conduction equation are considered, featuring boundary conditions of the first kind and of the third kind. Spectral problems for the difference and differential operators are solved. We also present analytical and numerical results for four related problems: boundary value problems for ordinary differential equations (arising in the stationary case) and initial-boundary value problems for the standard heat transfer process, wave and hyperbolic heat conduction equations. The advantages of the FDSES method for the case of the first kind boundary conditions are demonstrated by comparison with FDS for several numerical examples. Some examples and numerical experiments are presented. Numerical solutions in the time for these problems are obtained by the MATLAB solver "ode15s".

\section{Mathematical Models}

We consider the following 1-D hyperbolic heat conduction problem in a plate:

$$
\left\{\begin{array}{l}
\tau \frac{\partial^{2} T(x, t)}{\partial t^{2}}+\frac{\partial T(x, t)}{\partial t}=\frac{\partial}{\partial x}\left(\bar{k} \frac{\partial T(x, t)}{\partial x}\right)+f(x, t), \quad x \in(0, L), t \in\left(0, t_{f}\right) \\
\frac{\partial T(0, t)}{\partial x}-\alpha_{0}\left(T(0, t)-T_{l}\right)=0, \frac{\partial T(L, t)}{\partial x}+\alpha_{1}\left(T(L, t)-T_{r}\right)=0, t \in\left(0, t_{f}\right) \\
T(x, 0)=T_{0}(x), \quad \frac{\partial T(x, 0)}{\partial t}=V_{0}(x), \quad x \in(0, L)
\end{array}\right.
$$

where $\bar{k}$ is the heat conductivity, $t_{f}$ is the final time, $\tau$ is the relaxation time $(\tau<1), T_{l}, T_{r}, T_{0}(x), f(x, t)$ are given temperatures and a source function, $\alpha_{0}, \alpha_{1}$ are the heat transfer coefficients (for boundary conditions of the first kind $\left.\alpha_{0}=\alpha_{1}=\infty\right)$.

In a sphere with a hole defined in the spherical coordinates by $0<r_{0}<r<$ $R$, assuming radial symmetry, with homogeneous boundary conditions (BC) 
we obtain the following 1-D hyperbolic heat conduction problem:

$$
\left\{\begin{array}{l}
\tau \frac{\partial^{2} T(r, t)}{\partial t^{2}}+\frac{\partial T(r, t)}{\partial t}=\frac{\bar{k}}{r} \frac{\partial^{2}(r T(r, t))}{\partial r^{2}}+f(r, t), \quad r \in\left(r_{0}, R\right), t \in\left(0, t_{f}\right), \\
\frac{\partial T\left(r_{0}, t\right)}{\partial r}-\alpha_{0} T\left(r_{0}, t\right)=0, \quad \frac{\partial T(R, t)}{\partial r}+\alpha_{1} T(R, t)=0, \quad t \in\left(0, t_{f}\right), \\
T(r, 0)=T_{0}(r), \quad \frac{\partial T(r, 0)}{\partial t}=V_{0}(r), \quad r \in\left(r_{0}, R\right),
\end{array}\right.
$$

where $r$ is the polar radius, $R, r_{0}$ are the radius of the sphere and hole. The transformation $V=T r, x=r-r_{0}$ reduces the problem (2.2) to the following hyperbolic heat conduction problem in the plate:

$$
\left\{\begin{array}{l}
\tau \frac{\partial^{2} V(x, t)}{\partial t^{2}}+\frac{\partial V(x, t)}{\partial t}=\frac{\partial}{\partial x}\left(\bar{k} \frac{\partial V(x, t)}{\partial x}\right)+f\left(x+r_{0}, t\right), x \in(0, L), t \in\left(0, t_{f}\right), \\
\frac{\partial V(0, t)}{\partial x}-\sigma_{1} V(0, t)=0, \quad \frac{\partial V(L, t)}{\partial x}+\sigma_{2} V(L, t)=0, \quad t \in\left(0, t_{f}\right), \\
V(x, 0)=T_{0}\left(x+r_{0}\right)\left(x+r_{0}\right), \quad \frac{\partial V(x, 0)}{\partial t}=V_{0}\left(x+r_{0}\right)\left(x+r_{0}\right), \quad x \in(0, L)
\end{array}\right.
$$

where $\sigma_{1}=\alpha_{0}+1 / r_{0}, \sigma_{2}=\alpha_{1}-1 / R \geq 0, T(r, t)=V\left(x+r_{0}, t\right) /\left(x+r_{0}\right)$, $L=R-r_{0}$. In the case of a sphere $\left(r_{0} \rightarrow 0\right)$ the first boundary condition is $V(0, t)=0$ or $\sigma_{1}=\infty$ and $T(0, t)=\frac{\partial V(0, t)}{\partial x}$.

\section{Solution of the Problem (2.1) with Homogeneous BC of the First Kind}

If the surrounding temperature $T_{l}, T_{r}$ in (2.1) is constant and $\alpha_{0}=\alpha_{1}=\infty$ then using the transformation

$$
V(x, t)=T(x, t)-T_{h}(x), \quad T_{h}(x)=\left(x T_{r}+(L-x) T_{l}\right) / L
$$

we obtain a problem with homogeneous BCs of the first kind and $V(x, 0)=$ $V^{0}(x)=T_{0}(x)-T_{h}(x)$

We consider the uniform grid in the space $x_{k}=k h, k=\overline{0, N}, N h=L$. Using the standard finite difference approximation for partial derivatives of the second order with respect to $x$, we obtain an initial value problem for the system of ordinary differential equations (ODEs) of the second order:

$$
\left\{\begin{array}{l}
\tau \ddot{U}(t)+\dot{U}(t)+\bar{k} A U(t)=F(t), \\
U(0)=U_{0}, \quad \dot{U}(0)=V_{0},
\end{array}\right.
$$

where $A$ is the standard 3-diagonal matrix of order $M=N-1$ with the elements $\frac{4}{h^{2}}\{-1 ; 2 ;-1\}, U(t), \dot{U}(t), \ddot{U}(t), U_{0}, V_{0}, F(t)$ are the column-vectors of order $M$ with the elements $u_{k}(t) \approx V\left(x_{k}, t\right), \dot{u}_{k}(t) \approx \frac{\partial V\left(x_{k}, t\right)}{\partial t}, \ddot{u}_{k}(t) \approx \frac{\partial^{2} V\left(x_{k}, t\right)}{\partial t^{2}}$, $u_{k}(0)=V\left(x_{k}, 0\right), v_{k}(0)=V_{0}\left(x_{k}\right), f\left(x_{k}, t\right), k=\overline{1, M}$.

The corresponding discrete spectral problem

$$
A p^{k}=\mu_{k} p^{k}, \quad k=1, \ldots, M
$$


has the following solution [6]: eigenvalues $\mu_{k}=\frac{4}{h^{2}} \sin ^{2} \frac{k \pi}{2 N}$ and the orthonormal eigenvectors $p^{k}$ with elements $p_{i}^{k}=\sqrt{\frac{2}{N}} \sin \frac{\pi i k}{N}, i, k=\overline{1, M}$.

From the spectral problem $A P=P D$ it follows that the matrix $A$ is represented in the form $A=P D P$, where $P=P^{-1}$ is the symmetric orthogonal matrix with the columns being the eigenvectors of $A$, elementwise $p_{i, k}=p_{i}^{k}$, $i, k=\overline{1, M}$, and $D$ is the diagonal matrix with the elements $d_{k, k}=\mu_{k}$, $k=\overline{1, M}$.

The solution of the spectral problem for the corresponding continuous (differential) problem

$$
-p^{\prime \prime}(x)=\lambda p(x), \quad p(0)=p(L)=0
$$

is $p_{k}(x)=\sqrt{\frac{2}{L}} \sin \frac{k \pi x}{L}, \lambda_{k}=\left(\frac{k \pi}{L}\right)^{2},\left(p_{k}, p_{m}\right)=\int_{0}^{L} p_{k}(x) p_{m}(x) d x=\delta_{k, m}$, where $\delta_{k, m}$ is the Kronecker delta. For the discrete problem the integral in the scalar product $\left(p_{k}, p_{m}\right)$ is approximated by the trapezoidal formula.

The difference scheme with the exact spectrum (FDSES) is obtained by replacing $A$ with $\tilde{A}=P \tilde{D} P$, where $\tilde{D}$ is the diagonal matrix with the first $M$ eigenvalues $\lambda_{k}$ of the differential operator $\left(-\frac{\partial^{2}}{\partial x^{2}}\right)$ as elements, i.e. $\tilde{d}_{k, k}=\lambda_{k}$. Note that $\tilde{A}$ is a full matrix.

The FDSES method is more stable than the method of finite difference approximation with central difference (FDS), because the eigenvalues are larger $\lambda_{k}>\mu_{k}$. We can construct analytical solutions of (3.1) using the spectral representation of matrix $\mathrm{A}$ in the form $A=P D P$. The transformation $W=P U$ decouples the system of ODEs

$$
\left\{\begin{array}{l}
\tau \ddot{W}(t)+\dot{W}(t)+\bar{k} D W(t)=G(t), \\
W(0)=P U_{0}, \quad \dot{W}(0)=P V_{0},
\end{array}\right.
$$

where $W(t), \dot{W}(t), \ddot{W}(t), W_{0}, \dot{W}(0), G(t)=P F(t)$ are the column-vectors of order $M$ with the elements $w_{k}(t), \dot{w}_{k}(t), \ddot{w}_{k}(t), w_{k}(0), \dot{w}_{k}(0), g_{k}(t), k=\overline{1, M}$. The solution of this system is given by

$$
\begin{aligned}
w_{k}(t)= & \exp (-0.5 t / \tau)\left[\frac{\sinh \left(\kappa_{k} t\right)}{\kappa_{k}}\left(\dot{w}_{k}(0)+0.5 w_{k}(0) / \tau\right)+\cosh \left(\kappa_{k} t\right) w_{k}(0)\right] \\
& +\frac{1}{\kappa_{k} \tau} \int_{0}^{t} \exp \left(-\frac{0.5}{\tau}(t-\xi)\right) \sinh \left(\kappa_{k}(t-\xi)\right) g_{k}(\xi) d \xi
\end{aligned}
$$

where $\kappa_{k}=\sqrt{0.25 / \tau^{2}-\bar{k} d_{k} / \tau}$. If $4 \bar{k} d_{k} \tau>1$, then the hyperbolic functions are replaced with the trigonometrical and the parameter $\kappa_{k}$ with $\sqrt{k d_{k} / \tau-0.25 / \tau^{2}}$. If $\kappa_{k}=0$, then we have

$$
\begin{aligned}
w_{k}(t) & =\exp (-0.5 t / \tau)\left[t\left(\dot{w}_{k}(0)+0.5 w_{k}(0) / \tau\right)+w_{k}(0)\right] \\
& +\frac{1}{\tau} \int_{0}^{t} \exp \left(-\frac{0.5}{\tau}(t-\xi)\right)(t-\xi) g_{k}(\xi) d \xi
\end{aligned}
$$

We can also construct analytical solutions by using the Fourier method in the following form: $V(x, t)=\sum_{k=1}^{\infty} w_{k}(t) p_{k}(x)$, where $p_{k}(x)$ are the or- 
thonormal eigenfunctions for the differential operator $\left(-\frac{\partial^{2}}{\partial x^{2}}\right)$ with homogeneous boundary conditions, $w_{k}(t)$ is the solution of $(3.3)$, with

$$
w_{k}(0)=\left(V^{0}, p_{k}\right), \quad \dot{w}_{k}(0)=\left(V_{0}, p_{k}\right), \quad g_{k}(t)=\left(F(t), p_{k} .\right.
$$

For a given function $q(x) \in C^{n}[0, L]$ the Fourier coefficients $b_{k}$ can be estimated as $b_{k}=\left(q, p_{k}\right)=\mathcal{O}\left(k^{-n-1}\right), k \rightarrow \infty$. Then for the discrete expressions

$$
\begin{aligned}
& q\left(x_{i}\right)=\sum_{k=1}^{M} a_{k} p_{k}\left(x_{i}\right), \quad i=1, \ldots, M, \quad p_{j N}\left(x_{i}\right)=0, \quad j=1,2,3, \ldots, \\
& a_{k}=\left[q, p_{k}\right]=h \sum_{i=1}^{M} q\left(x_{i}\right) p_{k}\left(x_{i}\right), \quad\left[p_{k}, p_{m}\right]=\delta_{k, m}
\end{aligned}
$$

it follows that $q\left(x_{i}\right)=\sum_{k=1}^{\infty} b_{k} p_{k}\left(x_{i}\right)$,

$$
\begin{aligned}
a_{m} & =\left[q, p_{m}\right]=\sum_{k=1}^{\infty} b_{k}\left[p_{k}, p_{m}\right] \\
& =b_{m}+\sum_{k=N+1}^{\infty} b_{k}\left[p_{k}, p_{m}\right]=b_{m}+\sum_{j=1}^{\infty} b_{j N+m}, \quad 1 \leq m \leq M .
\end{aligned}
$$

Then $a_{m}-b_{m}=\sum_{j=1}^{\infty} b_{j N+m}=\mathcal{O}\left((N+m)^{-n}\right), m=\overline{1, M}, N \rightarrow \infty$.

From $\mu_{k} \rightarrow \lambda_{k}(k \rightarrow \infty)$ it follows that for differentiable functions $V^{0}(x)$, $V_{0}(x), f(x, t)$ we have the convergence of the coefficients $w_{k}(0), \dot{w}_{k}(0), g_{k}(t)$ (3.3) and $w_{k}(t)$ to the Fourier coefficients for every time moment $t$ as $N \rightarrow \infty$. If $d_{k}=\lambda_{k}$, then $a_{m}=b_{m}, m=1, \ldots, M$.

\section{The Spectral Problems for Solving (2.3)}

From (2.3) we obtain the initial value problem for the system of ODEs of the second order in the matrix form (3.1), where $A$ is the 3 -diagonal matrix of the order $M=N+1$ in the form

$$
A=\frac{1}{h^{2}}\left(\begin{array}{ccccccc}
2+2 h \sigma_{1} & -2 & 0 & \ldots & 0 & 0 & 0 \\
-1 & 2 & -1 & \ldots & 0 & 0 & 0 \\
\ldots & \ldots & \ldots & \ldots & \ldots & \ldots & \\
0 & 0 & 0 & \ldots & -1 & 2 & -1 \\
0 & 0 & 0 & \ldots & 0 & -2 & 2+2 h \sigma_{2}
\end{array}\right)
$$

The 3-diagonal matrix $A$ can be represented by the difference operator [6]

$$
A y=\left\{\begin{array}{l}
-2\left(y_{1}-y_{0}\right) / h^{2}+2 \sigma_{1} y_{0} / h, \quad k=0 \\
-\left(y_{k+1}-2 y_{k}+y_{k-1}\right) / h^{2}, \quad k=1, \ldots, N-1 \\
-2\left(y_{N-1}-y_{N}\right) / h^{2}+2 \sigma_{2} y_{N} / h, \quad k=N
\end{array}\right.
$$

Using the scalar product of two vectors $\left[y^{1}, y^{2}\right]=h\left(\sum_{k=1}^{N-1} y_{k}^{1} y_{k}^{2}+0.5\left(y_{0}^{1} y_{0}^{2}+\right.\right.$ $\left.\left.y_{N}^{1} y_{N}^{2}\right)\right)$ it can be proved, that the operator $A$ is symmetric and $[A y, y] \geq 0[6]$. 
The solution of the corresponding spectral problem

$$
-y^{\prime \prime}(x)=\lambda^{2} y(x), x \in(0, L), y^{\prime}(0)-\sigma_{1} y(0)=0, y^{\prime}(L)+\sigma_{2} y(L)=0,
$$

can be given in the following form:

$$
\left\{\begin{array}{l}
y_{n}(x)=C_{n}^{-1}\left(\lambda_{n} \cos \left(\lambda_{n} x\right)+\sigma_{1} \sin \left(\lambda_{n} x\right)\right), \\
C_{n}^{2}=0.5\left(L\left(\lambda_{n}^{2}+\sigma_{1}^{2}\right)+\frac{\sigma_{2}\left(\lambda_{n}^{2}+\sigma_{1}^{2}\right)}{\lambda_{n}^{2}+\sigma_{2}^{2}}+\sigma_{1}\right),
\end{array}\right.
$$

where $\left(y_{n}, y_{m}\right)=\int_{0}^{L} y_{n}(x) y_{m}(x) d x=\delta_{n, m}$ and $\lambda_{n}$ are the positive roots of the transcendental equation:

$$
\cot \left(\lambda_{n} L\right)=\frac{\lambda_{n}}{\sigma_{1}+\sigma_{2}}-\frac{\sigma_{1} \sigma_{2}}{\lambda_{n}\left(\sigma_{1}+\sigma_{2}\right)}, \quad n=1,2,3, \ldots
$$

The discrete spectral problem $A y^{n}=\mu_{n} y^{n}, n=1, \ldots, N+1$ has the solution [6]

$$
\left\{\begin{array}{l}
y_{j}^{n}=C_{n}^{-1}\left(\frac{\sin \left(p_{n} h\right)}{h} \cos \left(p_{n} x_{j}\right)+\sigma_{1} \sin \left(p_{n} x_{j}\right)\right), \quad j=0, \ldots, N, \\
\mu_{n}=\frac{4}{h^{2}} \sin ^{2}\left(p_{n} h / 2\right),
\end{array}\right.
$$

where $p_{n}$ are the positive roots of the following transcendental equation

$$
\cot \left(p_{n} L\right)=\frac{\sin ^{2}\left(p_{n} h\right)-h^{2} \sigma_{1} \sigma_{2}}{h\left(\sigma_{1}+\sigma_{2}\right) \sin \left(p_{n} h\right)}, \quad n=\overline{1, N+1} .
$$

The constants $C_{n}^{2}=\left[y^{n}, y^{n}\right]$ can be obtained in the following form

$$
\begin{aligned}
C_{n}^{2}= & 0.5 L\left(\sigma_{1}^{2}+\frac{\sin ^{2}\left(p_{n} h\right)}{h^{2}}\right)+\frac{\sin \left(2 p_{n} L\right) \sin \left(2 p_{n} h\right)}{8 h}-\sigma_{1}^{2} \frac{h \sin \left(2 p_{n} L\right) \cos \left(p_{n} h\right)}{4 \sin \left(p_{n} h\right)} \\
& +\sigma_{1} \sin ^{2}\left(p_{n} L\right) \cos \left(p_{n} h\right) .
\end{aligned}
$$

In the limit case it follows that $\mu_{n} \rightarrow \lambda_{n}^{2}$ as $h \rightarrow 0$.

The experiments with MATLAB (the spectral problem is solved with MATLAB solver "eig") show that the first roots $p_{n}, n=\overline{N-1}$ belong to the interval $((n-1) \pi, n \pi), \mu_{n} \leq \frac{4}{h^{2}}$ and can be obtained from (4.2), but the two last roots $p_{N}, p_{N+1}$ can not be obtained from this transcendental equation $\left(\mu_{n} \geq \frac{4}{h^{2}}\right.$, $n=N, N+1)$. In this case special solutions with $\mu \geq \frac{4}{h^{2}}$ can be obtained in the form $\mu=\frac{4}{h^{2}} \cosh ^{2} \frac{p h}{2}$. Then $y_{j}=(-1)^{j}\left[C_{1} \sinh \left(p x_{j}\right)+C_{2} \cosh \left(p x_{j}\right)\right]$. The constants $C_{1}, C_{2}$ are determined from the difference equations by $j=0, j=N$ and the values $p_{N}, p_{N+1}$ are obtained from the new transcendental equation. Depending on the fixed parameter $Q=L \sigma_{1} \sigma_{2} /\left(\sigma_{1}+\sigma_{2}\right)$ we can obtain one $(Q<1)$ or two $(Q \geq 1)$ roots from the following transcendental equation:

$$
\operatorname{coth}\left(p_{n} L\right)=\frac{\sinh ^{2}\left(p_{n} h\right)+h^{2} \sigma_{1} \sigma_{2}}{h\left(\sigma_{1}+\sigma_{2}\right) \sinh \left(p_{n} h\right)}, \quad n=\overline{N, N+1} .
$$

The expression $Q=1$ is obtained from this equation in the limit case when $p_{n} \rightarrow 0$. 
The corresponding eigenvalues and orthonormal eigenvectors are

$$
\left\{\begin{array}{l}
y_{j}^{n}=C_{n}^{-1}(-1)^{j}\left(\frac{\sinh \left(p_{n} h\right)}{h} \cosh \left(p_{n} x_{j}\right)-\sigma_{1} \sinh \left(p_{n} x_{j}\right)\right), \quad j=\overline{0, N}, \\
\mu_{n}=\frac{4}{h^{2}} \cosh ^{2}\left(p_{n} h / 2\right) .
\end{array}\right.
$$

The constants $C_{n}^{2}$ in this case are given by

$$
\left\{\begin{aligned}
C_{n}^{2}= & 0.5 L\left(-\sigma_{1}^{2}+\frac{\sinh ^{2}\left(p_{n} h\right)}{h^{2}}\right)+\frac{\sinh \left(2 p_{n} L\right) \sin h\left(2 p_{n} h\right)}{8 h} \\
& +\sigma_{1}^{2} \frac{h \sinh \left(2 p_{n} L\right) \cosh \left(p_{n} h\right)}{4 \sinh \left(p_{n} h\right)}-\sigma_{1} \sinh ^{2}\left(p_{n} L\right) \cosh \left(p_{n} h\right) .
\end{aligned}\right.
$$

Then we have the orthonormal eigenvectors $y^{n}, y^{m}$ for all $n, m=\overline{1, N+1}$.

For $Q=1$ the single eigenvalue is $\mu_{N}=4 / h^{2},\left(p_{N}=0\right)$ and the corresponding components $y_{j}^{N}$ of the orthonormal eigenvector $y^{N}$ are obtained as the limit $\left(p_{N} \rightarrow 0\right)$ of the expression $y_{j}^{N} / C_{N}$ in the following form:

$$
y_{j}^{N}=(-1)^{j}\left(1-\sigma_{1} x_{j}\right) \sqrt{6 h /\left(6 L+2 \sigma_{1}^{2} L^{3}+L \sigma_{1}^{2} h^{2}-6 \sigma_{1} L^{2}\right)}, \quad j=\overline{0, N} .
$$

The spectral problem for $\sigma_{1}=0, \sigma_{2}=\alpha$ has the solution

$$
y_{j}^{n}=\frac{\cos \left(p_{n} x_{j}\right)}{C_{n}}, \quad C_{n}^{2}=\frac{1}{2}\left(L+\frac{h}{2} \frac{\sin \left(2 p_{n} L\right)}{\tan \left(p_{n} h\right)}\right), \quad \mu_{n}=\frac{4}{h^{2}} \sin ^{2}\left(p_{n} h / 2\right),
$$

where $p_{n}$ are the positive roots of the following transcendental equation:

$$
\tan \left(p_{n} L\right) \sin \left(p_{n} h\right) / h=\alpha, \quad n=1, \ldots, N .
$$

For $n=N+1$ we have a special solution of the spectral problem

$$
y_{j}^{n}=\frac{\cosh \left(p_{n} x_{j}\right)}{C_{n}}, \quad C_{n}^{2}=\frac{1}{2}\left(L+\frac{h}{2} \frac{\sinh \left(2 p_{n} L\right)}{\tanh \left(p_{n} h\right)}\right), \quad \mu_{n}=\frac{4}{h^{2}} \cosh ^{2}\left(p_{n} h / 2\right),
$$

where $p_{n}$ is the positive root of the new transcendental equation

$$
\tanh \left(p_{n} L\right) \sinh \left(p_{n} h\right) / h=\alpha .
$$

Then we have orthonormal eigenvectors $y^{n}, y^{m}$, where the discrete scalar product is defines as $\left[y^{n}, y^{m}\right]=\delta_{n, m}$ for all $n, m=1, \ldots, N+1$.

The solution of the corresponding spectral problem for the differential equation

$$
-y^{\prime \prime}(x)=\lambda^{2} y(x), \quad x \in(0, L), \quad y^{\prime}(0)=0, \quad y^{\prime}(L)+\alpha y(L)=0,
$$

is given in following form

$$
y_{n}(x)=\frac{\cos \left(\lambda_{n} x\right)}{C_{n}}, \quad C_{n}^{2}=\frac{1}{2}\left(L+0.5 \sin \left(2 \lambda_{n}\right) / \lambda_{n}\right)=\frac{1}{2}\left(L+\frac{\alpha}{\alpha^{2}+\lambda_{n}^{2}}\right),
$$

where $\left(y_{n}, y_{m}\right)=\int_{0}^{L} y_{n}(x) y_{m}(x) d x=\delta_{n, m}$ and $\lambda_{n}$ are the positive roots of the transcendental equation $\tan \left(\lambda_{n} L\right) \lambda_{n}=\alpha, n=1,2,3, \ldots$ In the scalar product $\left[y_{n}, y_{m}\right]$ the integral $\left(y_{n}, y_{m}\right)$ is approximated using the trapezoidal formula. In the limit case, as $h \rightarrow 0$, we have $p_{n} \rightarrow \lambda_{n}$ for $n=1, \ldots, N$. 


\section{The Solution of the Problem (2.1) with Homogeneous $\mathrm{BC}$ of the Third Kind}

Similarly we obtain from (2.1) for $T_{l}=T_{r}=0$ the initial value problem for the system of ODEs (3.1), where the order of column-vectors is $M=N+1$. The matrix $A$ can be represented in the form $A=P D P^{T}$, where the columns of the matrix $P$ and the diagonal matrix $D$ contain $M$ orthonormal eigenvectors $y^{n}$ and eigenvalues $\mu_{n}, n=\overline{1, M}$. From $P^{T} P=E$ it follows that $P^{-1}=P^{T}$.

We can also use the Fourier method in the form $T(x, t)=\sum_{k=1}^{\infty} w_{k}(t) y_{k}(x)$, where $y_{k}(x)$ are the orthonormal eigenfunctions, $w_{k}(t)$ is the solution (3.3), with $w_{k}(0)=\left(T_{0}, y_{k}\right), \dot{w}_{k}(0)=\left(V_{0}, y_{k}\right), g_{k}(t)=\left(F(t), y_{k}\right)$.

For the FDSES the matrix $A$ is represented in the form $A=P D P^{T}$, where the diagonal matrix $D$ contains the first $M$ eigenvalues $d_{n}=\lambda_{n}^{2}, n=1, \ldots, M$ of the differential operator $\left(-\frac{\partial^{2}}{\partial x^{2}}\right)$ respectively.

It is possible to replace the column of the matrix $P$ with the first eigenfunctions $y_{j}^{n}=y_{n}\left(x_{j}\right), n, j=\overline{1, M}$ of the continuous problem. Then the eigenvectors $\left(y^{n}, y^{m}\right)$ or column-vectors of the matrix $P$ is not orthogonal in the discrete scalar product $\left[y^{n}, y^{m}\right]\left(P^{T} P \neq E\right)$ and the analytic form (3.3) is not valid.

If the heat conductivity $\bar{k}(x)$ is a functions of $x$ then we can obtain the discrete eigenvectors and eigenvalues using the MATLAB operator $[P, D]=$ eig $(A)$, where $A$ is the matrix of the difference operator with second order approximation. The orthogonal eigenvectors $p^{n}$ should be normalized with respect to the scalar product $\sqrt{\left[y^{n}, y^{n}\right]}$. The eigenvalues of the differential problem can be obtained with the MATLAB solver "bvp4". However, this algorithm is of high computational complexity.

The FDSES method performs well for nonlinear problems, when the diffusion term in (2.1) is given in the form $\frac{\partial^{2}}{\partial x^{2}} \phi(T)$. Then for $\frac{d \phi(T)}{d T}=\bar{k}(x, t)$ :

$$
\frac{\partial^{2}}{\partial x^{2}} \phi(T)=\frac{\partial}{\partial x}\left(\bar{k}(x, t) \frac{\partial T(x, t)}{\partial x}\right) .
$$

In this case from (3.1) we have the nonlinear system of ODEs with the term $A \phi(U)$, where $\phi(U)=\left(\phi\left(u_{1}(t)\right), \phi\left(u_{2}(t)\right), \ldots, \phi\left(u_{M}(t)\right)\right)^{T}, A=P D P^{T}$. The nonlinear heat transfer equation with power functions $\phi(T)$ is considered in [3].

Similarly we can consider the analytical solutions of (3.1) using the spectral representation of matrix $\mathrm{A}$ in the form $A=P D P^{T}$. From the transformation $W=P^{T} U$ we obtain the decoupled system of ODEs $(3.2)$, where $W(0)=$ $P^{T} U_{0}, \dot{W}(0)=P^{T} V_{0}, G(t)=P^{T} F(t)$ are the column-vectors of $N+1$ order. The solution of this system is given in the form (3.3). In this case, in order to compute the scalar product the first and last components of vectors $U_{0}$, $V_{0}, F(t)$ are divided by $\sqrt{2}$, but $w_{1}(t), w_{N+1}(t)$ need to be multiplied by $\sqrt{2}$. Similarly we can consider the analytical solutions of $(2.3)$.

\section{Some Examples and Numerical Results}

Boundary value problems for ODE with the homogeneous BC of the first kind (stationary problem). In the stationary case from $(2.1)(\bar{k}=1)$ 
we have the boundary value problem with BCs of the first kind

$$
-V^{\prime \prime}(x)=f(x), \quad x \in(0, L), \quad V(0)=V(L)=0 .
$$

Then from (3.1) we have a system of linear algebraic equations $A U=F$, where $U, F$ are the column-vectors with the elements $u_{k} \approx V\left(x_{k}\right), f_{k}=f\left(x_{k}\right), k=$ $\overline{1, M}, M=N-1$. Using the transformation $W=P U$ or $U=P W$ we can decouple system (3.2) into the form $D W=G,(G=P F)$ with the solution $w_{k}=g_{k} / d_{k}$, where $g_{k}=(P f)_{k}=\sum_{j=1}^{M} p_{k, j} f_{j}$. Therefore $U=P D^{-1} P F$ or

$$
u_{i}=\sum_{k=1}^{M} p_{i, k} w_{k}=\sum_{k, j} \frac{p_{i, k}}{d_{k}} p_{k, j} f_{j}, \quad i=1, \ldots, M .
$$

We obtain FDS method by setting $d_{k}=\mu_{k}$ and FDSES method by $d_{k}=$ $\lambda_{k}$. For the Fourier method $V(x)=\sum_{k=1}^{\infty} w_{k} p_{k}(x)$, where $w_{k}=g_{k} / d_{k}, g_{k}=$ $\left(p_{k}, f\right)=\int_{0}^{L} p_{k}(x) f(x) d x$. If $f(x)=\sum_{k=1}^{K} a_{k} \sin \frac{\pi k x}{L}, K \leq M$, then FDSES and Fourier method with $M$ at least summands are exact methods, but FDS is a method of the second order approximation. In this case the solution is $V(x)=\sum_{k=1}^{K} \frac{a_{k}}{d_{k}} \sin \frac{\pi k x}{L}$. By $K=1, L=10$ the calculations with FDS using MATLAB give the following results for the maximal error: $0.5376(N=5)$, $0.1873(N=10), 0.06596(N=20)$.

If $f(x)=\sin \frac{\pi x}{L}+\frac{x}{L}\left(1-\frac{x}{L}\right)$ then $V(x)=\left(\frac{L}{\pi}\right)^{2} \sin \frac{\pi x}{L}-\frac{x^{3}}{6 L}+\frac{x^{4}}{12 L^{2}}+\frac{x L}{12}$ and we have the following errors: for FDS $0.6735(N=5), 0.2353(N=10)$, for FDSES $0.0029(N=5), 0.0003(N=10)$.

Boundary value problems for ODE with the homogeneous BC of the third kind. For finite value $\sigma_{1}>0, \sigma_{2}>0$ we consider the following stationary problem for ODEs:

$$
-T^{\prime \prime}(x)=f(x), \quad x \in(0, L), \quad T^{\prime}(0)-\sigma_{1} T(0)=0, \quad T^{\prime}(L)+\sigma_{2} T(L)=0 .
$$

The boundary value problem is solved by $f(x)=12 x^{2} C_{0}+\sigma_{1} \sin (x), C_{0}=$ $\left(\sigma_{1} \cos (L)+\sigma_{1} \sigma_{2} \sin L+\sigma_{2}\right) /\left(4 L^{3}+\sigma_{2} L^{4}\right)$. The exact solution of the differential problem is $u(x)=-x^{4} C_{0}+1+\sigma_{1} \sin (x)$.

The solution of the problem is obtained with MATLAB in the form $U=$ $A^{-1} F$ or $U=P D^{-1} P^{T} F$, where $D^{-1}$ is the diagonal matrix with elements $d_{k}^{-1}, k=\overline{1, M}, M=N+1$. In this case the first and last components of vector $F$ are divided with $\sqrt{2}$, but elements of the solution vector $U u_{1}, u_{N+1}$ are multiplied by $\sqrt{2}$. In the Table 1 we can see the last eigenvalues $p_{N}, p_{N+1}, \mu_{N}$, $\mu_{N+1}$ obtained from (4.1), (4.2) and the maximum norm of the error of the finite difference scheme $(\delta(F D S))$ and FDSES $(\delta(F D S E S))$.

Linear heat transfer equation. The analytical solution of the heat transfer problem (2.1) with $\bar{k}=L=1, T_{l}=T_{r}=f=\tau=0, T_{0}=1, \alpha_{0}=\alpha_{1}=\infty$ (the first kind homogeneous BC) with discontinuous initial and boundary data can be obtained from the following Fourier series:

$$
T(x, t)=\frac{4}{\pi} \sum_{i=0}^{\infty} \frac{1}{2 i+1} \exp \left(-(2 i+1)^{2} \pi^{2} t\right) \sin ((2 i+1) \pi x) .
$$


Table 1. The values of $\sigma_{1}, \sigma_{2}, N, L, \delta(F D S), \delta(F D S E S), p_{N}, \mu_{N}, p_{N+1}, \mu_{N+1}$.

\begin{tabular}{llllllllll}
\hline$\sigma_{1}$ & $\sigma_{2}$ & $N$ & $L$ & $\delta(F D S)$ & $\delta(F D S E S)$ & $p_{N}$ & $\mu_{N}$ & $p_{N+1}$ & $\mu_{N+1}$ \\
\hline 2 & 0.1 & 10 & 11.0 & 0.4795 & 0.1788 & 0.037 & 3.3072 & 1.3906 & 5.6473 \\
2 & 0.1 & 20 & 11.0 & 0.1582 & 0.0525 & 0.037 & 13.225 & 1.7297 & 16.440 \\
2 & 0.1 & 15 & 11.0 & 0.2484 & 0.0856 & 0.037 & 7.4394 & 1.6038 & 10.321 \\
1 & 2.0 & 10 & 2.0 & 0.0099 & 0.0054 & 0.840 & 100.70 & 1.950 & 103.87 \\
1 & 2.0 & 15 & 2.0 & 0.0052 & 0.0027 & 0.840 & 225.71 & 1.950 & 228.95 \\
1 & 2.0 & 30 & 2.0 & 0.0018 & 0.0009 & 0.840 & 900.70 & 1.950 & 904.00 \\
1 & 2.0 & 60 & 2.0 & 0.0006 & 0.0003 & 0.840 & 3600.7 & 1.950 & 3604.0 \\
0 & 1.0 & 10 & 2.0 & 0.0053 & 0.0036 & 14.48 & 98.500 & 1.030 & 101.06 \\
\hline
\end{tabular}

The corresponding solution of (3.1) with FDS is given in the form $U(t)=$ $P \exp (D t) P U_{0}$, where $U_{0}$ is the column vector with ones, the diagonal matrix $D$ contains the discrete eigenvalues $\mu_{k}, k=1, \ldots, N-1$. For the FDSES the elements of matrix $D$ are replaced with the first $N-1$ eigenvalues $\lambda_{k}$ of the differential operator. The maximal error for $t=0.02, N=10$ is 0.089 for FDS and 0.0102 for FDSES. The results obtained with the Fourier series contain oscillations near $x=0, x=L$ due to the Gibbs phenomenon. For the FDSES method these oscillations are damped.

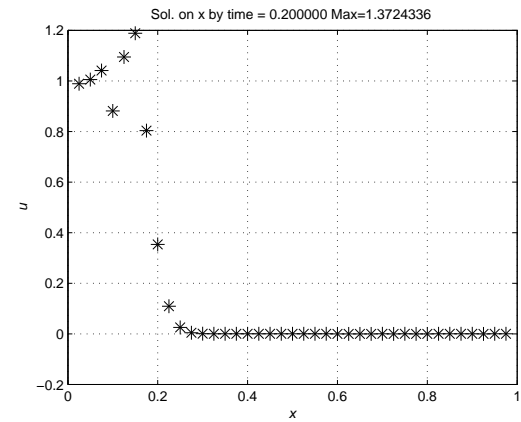

Figure 1. FDS solution depending on $x$ for $N=40, t=0.2$.

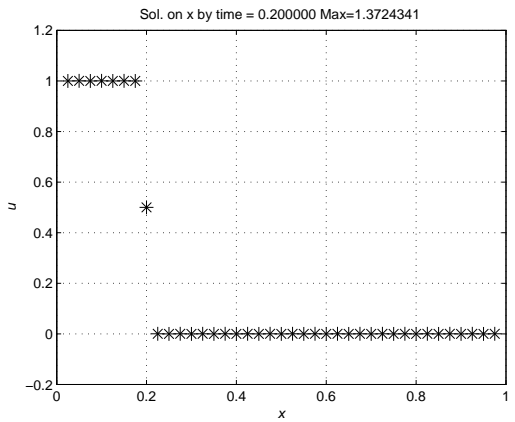

Figure 2. FDSES solution depending on $x$ for $N=40, t=0.2$.

Linear wave equation. For numerical calculation we consider the test problem for the standard wave equation (2.1) when the parabolic term $\frac{\partial T(x, t)}{\partial t}$ is excluded from the equation. The following data and parameters are used in computations: $\tau=1, \alpha_{0}=\alpha_{1}=\infty, T_{l}=1, T_{r}=0, f=T_{0}=V_{0}=0$. Using the transformation $V(x, t)=T(x, t)-1+x$ for the function $V(x, t)$ we get the problem with the homogeneous BC, where $V(x, 0)=x-1, \frac{\partial V(x, 0)}{\partial t}=0$. The numerical results for $(N=40, t=0.2)$ are presented in Figs. 1, 2.

Problem for heat conduction equation with discontinuous conditions. The numerical experiments for the problem (2.1) with $L=\bar{k}=1, \tau=0.1, t_{f}=$ 


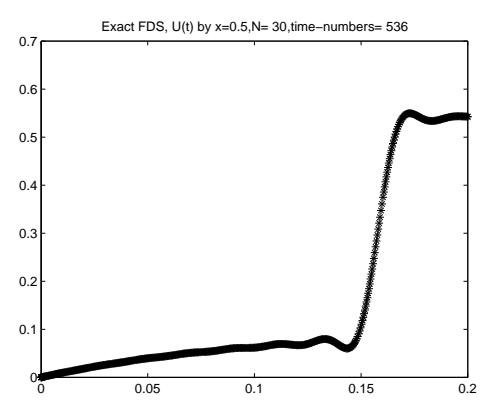

Figure 3. Solution of FDSES $T(0.5, t)$ for $N=30$.

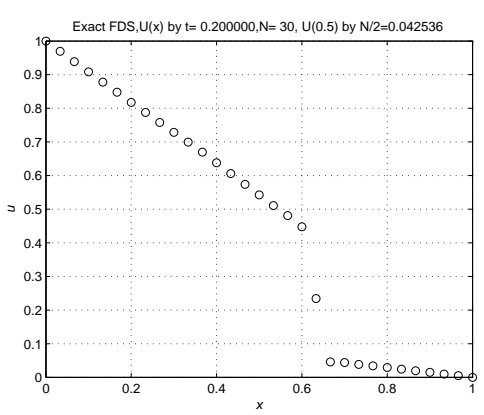

Figure 4. Solution $T(x, 0.2)$ with FDSES and $N=30$.

$0.2, T_{l}=1, T_{r}=0, T_{0}=0, f=V_{0}=0$ (the initial and boundary conditions are discontinuous [1]) are performed with the MATLAB solver "ode15s". The graphics of numerical results obtained with FDSES for $N=30$ are presented in Figs. 3, 4. In Figs. 5, 6 we present results computed by using the Fourier method series

$$
V(x, t)=-2 \exp (-0.5 t / \tau) \sum_{k=1}^{\infty} \frac{\sin (k \pi x)}{k \pi}\left(\cosh \left(\kappa_{k} t\right)+\frac{\sinh \left(\kappa_{k} t\right)}{2 \kappa_{k} \tau}\right),
$$

where $w_{k}(0)=-\frac{\sqrt{2}}{\pi k}, \dot{w}_{k}(0)=0, N=80$. We note that the Gibbs effect is observed.

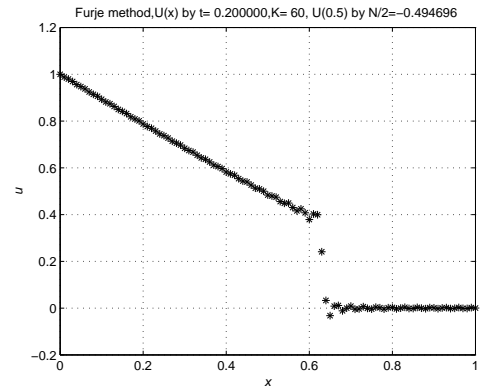

Figure 5. Solution $T(x, 0.2)$ with Fourier method, $N=80$.

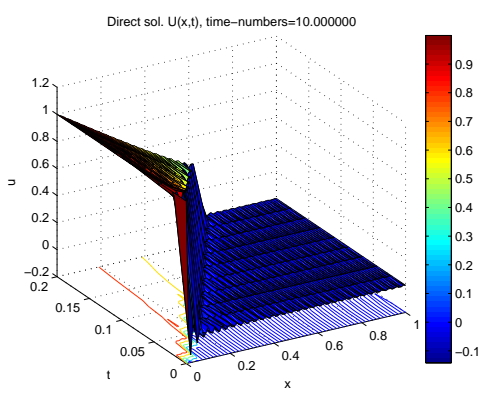

Figure 6. Solution $T(x, t)$ with Fourier method, $N=80$.

In Figs. 7, 8 we can see the results obtained with finite differences approximation and FDSES method with $N=200$.

Heat heat conduction equation for intensive steel quenching. The numerical results for the problem (2.1) $\left(\alpha_{0}=0, \alpha_{1}=\alpha, T_{l}=T_{r}=f=0\right)$ are 


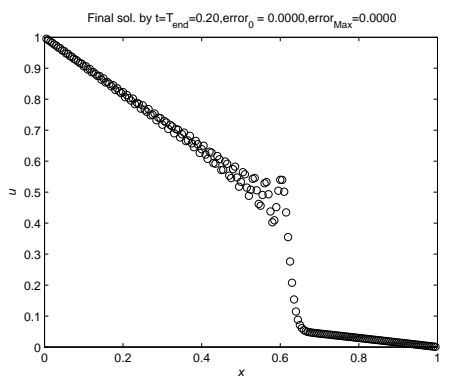

Figure 7. Solution $T(x, 0.2)$ with FDS, $N=200$.

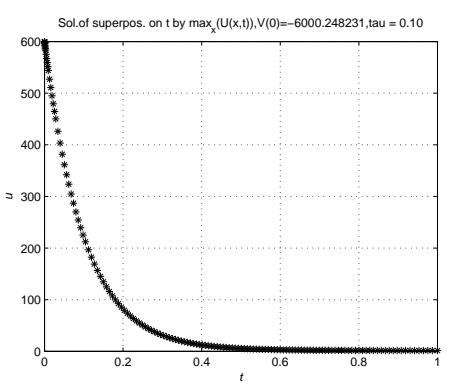

Figure 9. Solution $T(0.5, t)$ for $\tau=0.1$, $T_{0}(x)=600, V_{0}(x)=-6000.2$.

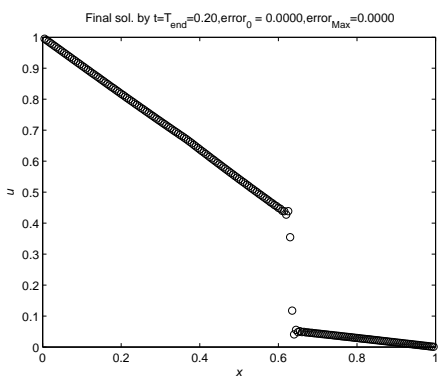

Figure 8. Solution $T(x, 0.2)$ with FDSES, $N=200$.

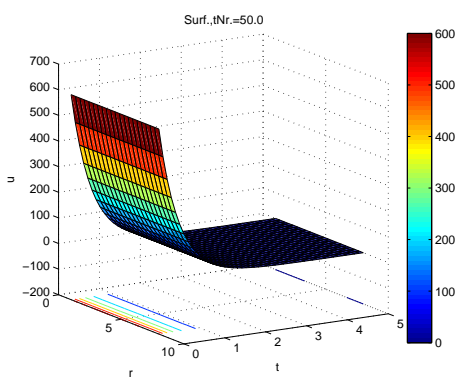

Figure 10. Solution depending on $t, r$ for $\tau=0.5, T_{0}(r)=600$, $V_{0}(r)=-1200.1$.

obtained for intensive steel quenching models with $\bar{k}=0.0001738602\left(\bar{k}=\frac{k}{c \rho}\right.$, $k=429 \frac{\mathrm{W}}{\mathrm{mC}}$ is the heat conductivity, $\rho=1.05 .10^{4} \frac{\mathrm{kg}}{\mathrm{mC}}$ is the density of steel, $c=235 \frac{\mathrm{J}}{\mathrm{kgC}}$ is the heat capacity), $\alpha=\frac{30}{429}$ and $L=1, \tau=0.1, T_{0}(x)=600$, $V_{0}=-6000.2, t_{f}=1, N=20$ (see, Fig. 9).

The numerical results for the problem (2.2) are obtained for the abovementioned parameters with $R=8, r_{0}=1, \tau=0.5, T_{0}(r)=600, V_{0}=-1200.1$, $t_{f}=5, N=20$ (see, Fig. 10).

\section{Conclusions}

- The hyperbolic heat conduction problems can be numerically modelled using FDS and FDSES methods.

- The advantages of the FDSES method for the problem with constant heat conductivity and with the first kind boundary conditions (BC) are demonstrated via several numerical examples in comparison with the FDS method. 
- In the case of the $\mathrm{BC}$ of the third kind the eigenvectors of the differential and discrete problems are different and the FDSES is obtained by using the orthonormal eigenvectors of the discrete problem.

- For nonlinear diffusion term $\frac{\partial^{2}}{\partial x^{2}} \phi(T)$ it is possible to obtain the algorithm of FDSES by numerical solutions of a system of nonlinear ODEs.

- For the discrete Fourier method numerical oscillations are present, when the initial and boundary conditions are discontinuous. For larger $N$ these oscillations disappear. The method of FDSES is free of oscillations.

- For the discrete spectral problem new transcendental equation and eigenvectors are obtained. All eigenvectors for the finite difference operator are obtained analytically.

\section{References}

[1] R. Ciegis. Numerical solution of hyperbolic heat conduction equation. Math. Model. Anal., 14(1):11-24, 2009. Doi:10.3846/1392-6292.2009.14.11-24.

[2] R. Čiegis, A. Dementev and G. Jankevičiūtè. Numerical analysis of hyperbolic two temperature model. Lith. Math. Journal, 48(1):46-60, 2008. Doi:10.1007/s10986-008-0005-6.

[3] A. Gedroics, H. Kalis and I. Kangro. Numerical methods for solving some nonlinear heat transfer problems. Int. J. Pure Appl. Math., 57(4):467-484, 2009.

[4] N.I. Kobasko. Intensive Steel Quenching Methods, Hanbook "Theory and Technology of Quenching". Springer-Verlag, 1992.

[5] V.L. Makarov and I.P. Gavrilyuk. On constructing the difference net circuits with the exact spectrum. Dopov. Akad. Nauk Ukr. RSR, Ser. A, pp. 1077-1080, 1975. (in Ukrainian)

[6] A.A. Samarskij. Theory of Finite Difference Schemes. Moscow, Nauka, 1977. (in Russian) 